\title{
Yunnan Minority Eye Study Suggests That Ethnic Differences in Myopia Are Due to Different Environmental Exposures
}

\author{
Ian G. Morgan ${ }^{1}$ and Kathryn Rose ${ }^{2}$ \\ ${ }^{1}$ Research School of Biology, Australian National University, Canberra, Australia; ian.morgan@anu.edu.au
}

${ }^{2}$ Orthoptics, Graduate School of Health, University of Technology Sydney, Sydney, Australia

The article by Pan et al. ${ }^{1}$ in the May issue shows that the prevalence of myopia is very low in adults from two ethnic groups-Han Chinese and Yi (one of China's national minority groups) living in the same rural area in Yunnan Province. The very low prevalence of myopia in both groups shows that myopia is not inevitable in East Asian populations, but requires exposure to environmental risk factors. Education levels were low in the samples studied. Time spent outdoors as a child was assessed by questionnaire, and over $60 \%$ reported at least 3 hours a day outdoors, which is much higher than is reported by today's better-educated and highly myopic young adults in urban China, consistent with the idea that intensive education and limited time outdoors combine to produce myopia. Evidence of this kind should finally put the idea that East Asians are inevitably myopic to rest. A related idea is that even if myopia is not inevitable, East Asian genetic backgrounds may make people more susceptible to risk factors. However, even this idea does not seem to stand up. ${ }^{2}$ Data from Singapore show that the prevalence of myopia is high in young Chinese and Malay adults (of East Asian origin) and in Indians (of South Asian origin). ${ }^{3}$ The latter observation is crucial, because there is a much lower prevalence of myopia in India, ${ }^{4}$ suggesting that the intensive education and limited time outdoors common across ethnic groups in Singapore explain the common high prevalence rates. Overall, this evidence suggests that ethnic differences in the prevalence of myopia are more likely to be explained in terms of differences in environmental exposures in different locations, than in genetic terms. $^{2}$

\section{References}

1. Pan C-W, Chen Q, Sheng X, et al. Ethnic variations in myopia and ocular biometry among adults in a rural community in China: the Yunnan Minority Eye Studies. Invest Ophthalmol Vis Sci. 2015;56:3235-3241.

2. Morgan I, Rose K. How genetic is school myopia? Prog Retinal Eye Res. 2005;24:1-38.

3. Koh V, Yang A, Saw SM, et al. Differences in prevalence of refractive errors in young Asian males in Singapore between 1996-1997 and 2009-2010. Ophthalmic Epidemiol. 2014;21:247-255.

4. Saxena R, Vashist P, Tandon R, et al. Prevalence of myopia and its risk factors in urban school children in Delhi: the North India Myopia Study (NIM study). PLoS One. 2015;10:e117349. 